\title{
N-terminal pro-brain natriuretic peptide in systemic sclerosis patients: correlation with nailfold capillaroscopy findings
}

\author{
Alexandru Caraba', Andreea Munteanu', Daniela Jianu², DianaNicoara², \\ Flavia Corina Babalic ${ }^{1}$, DinuValentin Balanescu ${ }^{3}$, DenisaPredeteanu ${ }^{3}$ \\ ${ }^{1}$ Department of Internal Medicine, Division of Rheumatology, \\ "Victor Babes" University of Medicine and Pharmacy, Timisoara, Romania \\ 2Department of Medical Semiology, "Victor Babes" University of Medicine and Pharmacy, Timisoara, Romania \\ 3Department of Internal Medicine and Rheumatology, "Sfanta Maria" Clinical Hospital, \\ "Carol Davila" University of Medicine and Pharmacy, Bucharest, Romania
}

\begin{abstract}
Background. Pulmonary arterial hypertension (PAH) is an important cause of morbidity and mortality in patients with systemic sclerosis (SSc). This condition is diagnosed by cardiac Doppler ultrasonography, right-heart catheterization, or by serum levels of N-terminal pro-brain natriuretic peptide (NT-proBNP). The aim of this study was to assess the association between serum NT-proBNP and nailfold capillaroscopic patterns in SSc patients. We also analyzed the association between pulmonary function parameters, NT-proBNP, and nailfold capillary findings in patients diagnosed with SSc and PAH.

Material and methods. We retrospectively analyzed SSc patients and healthy controls from our institution between July 2016 - December 2018. We assessed by chart review: pulmonary artery systolic pressure (PASP), forced vital capacity (FVC), forced expiratory volume in $1 \mathrm{sec} /$ forced vital capacity ratio (FEV1/FVC ratio), the number of nailfold capillaries/mm and NT-proBNP. Statistical analyses were performed using the Student's t-test, ANOVA test and the Pearson's correlation.

Results. Seventeen patients with SSc and 17 healthy controls matched for age and gender were included. Among SSc patients, 13 had diffuse cutaneous SSc (dcSSc) and 4 patients had limited cutaneous SSc (IcSSc). PAH was identified in 10 SSc patients. In SSc patients, significant correlations have been identified between PASP and NT-proBNP $(r=0.9, p<0.0001)$, nailfold capillaries density and PASP $(r=-0.95, p<0.0001)$, and nailfold capillaries density and NT-proBNP $(r=-0.84, p<0.0001)$.

Conclusion. We suggest that in patients with SSc, NT-proBNP is significantly correlated with PASP and nailfold capillaroscopic findings.
\end{abstract}

Keywords: nailfold capillaroscopy, NT-proBNP, pulmonary hypertension, systemic sclerosis

\section{INTRODUCTION}

Systemic sclerosis (SSc) is a chronic autoimmune disease characterized by skin and visceral fibrosis, widespread obliterative vasculopathy, and cellular and humoral immunity abnormalities $(1,2)$. Vascular changes are initially subclinical, but later they become clinically-evident in the form of obliterative vasculopathy (3). SSc complications are common and may present as ischemic digital ulcers, scleroderma renal crisis, or pulmonary arterial hypertension (PAH) (2).

Nailfold capillaroscopy has emerged as a useful tool in the staging of the involvement of the micro- circulation in SSc patients. This test offers details about the disease severity, the degree of visceral involvement, and the appearance and progression of sclerodermic microangiopathy $(3,4,5)$. Cutolo et al. defined three patterns of microvascular involvement in SSc patients: early (few giant capillaries, few capillary micro-hemorrhages, no evident loss of capillaries, and a relatively well-preserved capillary distribution), active (frequent giant capillaries, frequent capillary micro-hemorrhages, moderate loss of capillaries, absence of or mildly ramified capillaries with slight disorganization of the capillary architecture), and late (irregular enlargement of the capillaries, 
almost absent giant capillaries and micro-hemorrhages, severe loss of capillaries with extensive avascular areas, ramified/bushy capillaries, and intense disorganization of the normal capillary array) $(6,7)$.

$\mathrm{PAH}$, a potentially life-threatening condition, is a late complication of SSc, usually appearing after 10 years of disease, especially in patients with limited cutaneous $\mathrm{SSc}(\mathrm{lcSSc})$. In contrast, in patients with diffuse cutaneous SSc (dcSSc), PAH develops earlier in the course of the disease, and is considered to be a complication of interstitial lung disease. The incidence of PAH is estimated to be between $7-15 \%$, having an aggressive course $(8,9)$. Risk factors associated with the development of PAH are: older age, more severe peripheral vascular disease, extensive avascular areas on nailfold capillaroscopy (reduced number of capillaries $/ \mathrm{mm}$ ), or presence of interstitial lung disease/pulmonary fibrosis $(10,11)$.

PAH is generally diagnosed by cardiac Doppler ultrasonography, or by right-heart catheterization, by assessing the following parameters: pulmonary artery pressure (systolic, diastolic, and mean), right atrial pressure, pulmonary wedge pressure, and right ventricle pressure. Biochemical biomarkers are also important in diagnosing PAH. One such marker is the N-terminal pro-brain natriuretic peptide (NT-proBNP). It is released into the blood following the cleavage of proBNP, while having a longer halflife, which makes it suitable for detection (12). Over the past few years, the link between nailfold capillary abnormalities and target organ damage has been investigated. Some studies revealed a negative correlation between the nailfold capillary abnormalities, expressed as nailfold capillaries density and severity of organ involvement $(13,14,15)$.

The primary objective of this study was to assess the variability of serum NT-proBNP depending on the nailfold capillaroscopic patterns in SSc patients. The secondary objectives were to assess the associations between pulmonary function parameters, NT-proBNP, and nailfold capillaroscopy findings in patients with SSc and PAH.

\section{MATERIAL AND METHODS}

\section{Patients}

Patients diagnosed with SSc at our institution between July 2016 - December 2018 along with an equal number of healthy controls matched for age and gender, were included. The diagnosis of SSc was established based on the 2013 ACR/EULAR Classi- fication Criteria for Systemic Sclerosis (16). We excluded patients with previous pulmonary diseases not associated with SSc, overlap syndromes, uncontrolled systemic hypertension, cardiac diseases with NYHA classes II, III and IV heart failure, coagulation disorders, current smokers, and pregnant or breastfeeding women. All the patients gave their informed consent. The study was approved by the Ethics Committee of the Victor Babes University of Medicine and Pharmacy, Timişoara, Romania.

We assessed the following pulmonary parameters: pulmonary artery systolic pressure (PASP) (using a Siemens Acuson X300 Ultrasound System with a 2,5 MHz transducer), forced vital capacity (FVC), forced expiratory volume in $1 \mathrm{sec} /$ forced vital capacity ratio (FEV1/FVC ratio) (Thor Medical Systems). PAH was defined as a mean pulmonary arterial pressure over $25 \mathrm{mmHg}$ at rest (corresponding to an estimated PASP over $40 \mathrm{mmHg}$ ) (17).

The density of nailfold capillaries $/ \mathrm{mm}$ was determined by nailfold capillaroscopy (USB Digital Microscope, 2.0 Mega Pixel Digital Camera). Before this test, the patients were seated in a room with a stable temperature of $20-22^{\circ} \mathrm{C}$ for at least $15 \mathrm{~min}$ utes, in order to avoid capillary vasoconstriction (which increases false positive rates for avascular areas). The $2^{\text {nd }}, 3^{\text {rd }}, 4^{\text {th }}$ and $5^{\text {th }}$ digits of both hands were examined. Giant capillaries, capillary hemorrhages, avascular areas, ramified/bushy capillaries, and capillary architecture were recorded.

Serum NT-proBNP (immunochemistry/chemiluminescent immunoassay) was the marker used to characterize the right ventricle dysfunction associated with PAH. Antinuclear, anti-topoisomerase, and anti-centromere antibodies were determined using indirect immunofluorescence (HELMED).

The same variables were analyzed in both groups of patients.

\section{Statistical analysis}

Data are presented as mean \pm standard deviation. Statistical analyses were performed using Microsoft Excel the Student's t-test, ANOVA test, and the Pearson's correlation. Differences were considered statistically significant at a p-value $<0.05$.

\section{RESULTS}

We included 17 SSc patients and 17 healthy controls. Baseline demographics are presented in Table 1. Among SSc patients, 13 (76.5\%) had diffuse cuta- 
TABLE 1. Baseline demographics of the studied population

\begin{tabular}{|l|l|l|}
\hline \multirow{2}{*}{ Parameter } & \multicolumn{2}{l|}{ Value (mean \pm standard deviation) } \\
\cline { 2 - 3 } & SSc patients & Controls \\
\hline $\begin{array}{l}\text { Sex }[\mathrm{n}(\%)] \\
\text { Males }\end{array}$ & 17 & 17 \\
Females & $5(29.41 \%)$ & $5(29.41 \%)$ \\
\hline Mean age (years) & $12(70.59 \%)$ & $12(70.59 \%)$ \\
\hline Mean length of SSc evolution (years) & $50.47 \pm 5.46$ & $52.18 \pm 5.72$ \\
\hline Mean length of Raynaud's phenomenon evolution (years) & $6.23 \pm 1.71$ & - \\
\hline The drugs used by SSc patients at the initial evaluation & $9.58 \pm 3.77$ & - \\
& Bosentan & - \\
& (2 patients) & \\
& Macicentan (1 patient) & \\
& Calcium channel blockers & \\
& (17 patients) & \\
& Cyclophosphamide (6 patients) & \\
& Methotrexate (4 patients) & \\
\hline
\end{tabular}

neous SSc (dcSSc), whereas $4(23.5 \%)$ patients had the limited form of the disease (lcSSc). Raynaud's phenomenon was present in all cases. In the SSc group, $3(17.6 \%)$ patients had early nailfold capillaroscopic pattern, 7 (41.2\%) had the active pattern, and $7(41.2 \%)$ had the late pattern. Antinuclear antibodies were identified in all patients. Among these antibodies, anti-topoisomerase I antibodies were identified in $13(76.5 \%)$ SSc patients, whereas anti-centromere in $4(23.5 \%)$ patients. PASP and serum NT-proBNP were significantly higher in SSc patients versus healthy controls, whereas FVC, FEV1/ FVC ratio, and capillary density/mm were significantly lower (Table 2). There were no statistically significant differences of NT-proBNP and PASP between controls and SSc without PAH.
PAH was identified in $10(58.9 \%) \mathrm{SSc}$ patients (8 patients with dcSSc and 2 patients with $1 \mathrm{cSSc}$ ). Clinical, pulmonary, and capillaroscopic parameters and NT-proBNP values in PAH related to SSc patients were presented in Table 3. In dcSSc patients, PAH was associated with interstitial lung disease/interstitial pulmonary fibrosis, expressed as reduced FVC, younger age, and lower mean length of SSc progression. In lcSSc, PAH appeared independently of the SSc related interstitial lung disease, mean age was higher, and mean SSc progression was longer than in patients with dcSSC.

Both NT-proBNP and PAH significantly increase with the severity of the capillaroscopic pattern (Table 4).

TABLE 2. Pulmonary and capillaroscopic parameters and NT-proBNP values in SSc patients and controls

\begin{tabular}{|l|c|c|c|}
\hline Parameter & SSc patients & Controls & p \\
\hline PASP $(\mathrm{mmHg})$ & $44.88 \pm 12.65$ & $29.82 \pm 1.55$ & $<\mathbf{0 . 0 0 1}$ \\
\hline NT-proBNP $(\mathrm{pg} / \mathrm{ml})$ & $196.11 \pm 91.78$ & $103.35 \pm 10.07$ & $<\mathbf{0 . 0 0 1}$ \\
\hline FVC $(\%)$ & $66.47 \pm 12.79$ & $84.82 \pm 3.16$ & $<\mathbf{0 . 0 0 1}$ \\
\hline FEV1/FVC (\%) & $82.58 \pm 4.34$ & $91.94 \pm 4.70$ & $<\mathbf{0 . 0 0 1}$ \\
\hline Capillary density/mm & $7.27 \pm 2.63$ & $11.57 \pm 1.04$ & $<\mathbf{0 . 0 0 1}$ \\
\hline
\end{tabular}

TABLE 3. Clinical, pulmonary, and capillaroscopic parameters and NT-proBNP values in $P A H$-SSc patients

\begin{tabular}{|l|c|c|c|}
\hline Parameter & dcSSc patients & IcSSc patients & p-value \\
\hline Mean age (years) & $48.5 \pm 5.09$ & $57 \pm 1.41$ & $<\mathbf{0 . 0 1}$ \\
\hline Mean length of SSc evolution (years) & $6 \pm 1.69$ & $8.5 \pm 0.70$ & $<\mathbf{0 . 0 5}$ \\
\hline PASP (mmHg) & $52.25 \pm 5.57$ & $62 \pm 8.48$ & 0.33 \\
\hline NT-proBNP (pg/ml) & $234.62 \pm 81.54$ & $302.5 \pm 122.32$ & 0.57 \\
\hline FVC (\%) & $54.75 \pm 4.94$ & $78.5 \pm 4.94$ & $<\mathbf{0 . 0 5}$ \\
\hline FEV1/FVC (\%) & $81.25 \pm 4.09$ & $80.5 \pm 0.70$ & 0.061 \\
\hline Capillaries density/mm & $\begin{array}{c}5.90 \pm 1.69 \\
\text { Active stage in 3 patients } \\
\text { Late stage in 5 patients }\end{array}$ & $\begin{array}{c}3.74 \pm 0.17 \\
\text { Late stage in 2 patients }\end{array}$ & $<0.01$ \\
\hline
\end{tabular}


TABLE 4. NT-proBNP and PASP values in SSc patients and controls

\begin{tabular}{|l|l|c|c|c|c|}
\hline \multirow{2}{*}{ Parameter } & \multirow{2}{*}{ Controls } & \multicolumn{2}{|l|}{ SSc patients with nailfold capillaroscopy stage } & \multirow{2}{*}{ p-value } \\
\cline { 3 - 5 } & & Early & Active & Late & \\
\hline NT-proBNP $(\mathrm{pg} / \mathrm{ml})$ & $103.35 \pm 10.07$ & $120 \pm 1.73$ & $143 \pm 25.33$ & $281.85 \pm 84$ & $<0.0001$ \\
\hline PASP $(\mathrm{mmHg})$ & $29.82 \pm 1.55$ & $30.33 \pm 0.57$ & $38.85 \pm 8$ & $57.14 \pm 6.28$ & $<0.0001$ \\
\hline
\end{tabular}

In SSc patients, significant correlations have been identified between PASP and NT-proBNP $(\mathrm{p}<0.0001)$, nailfold capillaries density and PASP $(\mathrm{p}<0.0001)$, and nailfold capillaries density and NT-proBNP $(\mathrm{p}<0.0001)$ (Figures 1, 2, 3).

\section{DISCUSSION}

Vascular disease, a major pathologic manifestation of SSc, mainly affects the microcirculation (18). $\mathrm{SSc}$ vascular involvement is an important contributor to the morbidity and mortality associated with this disease (19). Vascular dysfunction occurs early during the course of SSc, followed by disorganiza- tion of the microvascular architecture. This pathologic event may represent even the initial step of progressing towards systemic fibrotic disease and has a negative prognosis $(20,21,22)$.

Microvasculature changes are seen in all involved organs (i.e., lungs, heart, kidneys, and gastrointestinal tract), demonstrating the widespread nature of capillary changes in SSc (4). Some studies suggest that the nailfold capillaroscopic detection of microvascular lesions in SSc may precede clinically overt involvement of other tissues and organs $(13,15)$. At the same time, capillary loss detected by nailfold capillaroscopy has been associated with PAH, inter-

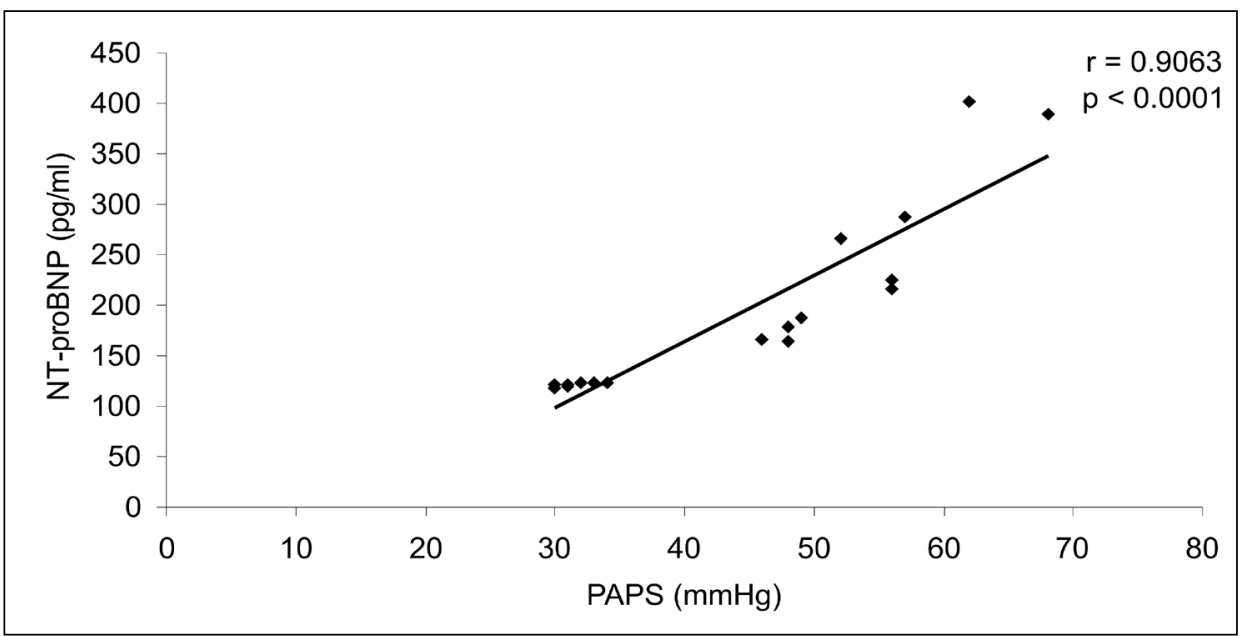

Figure 1. Pearson correlation between PASP and NT-proBNP in SSc patients

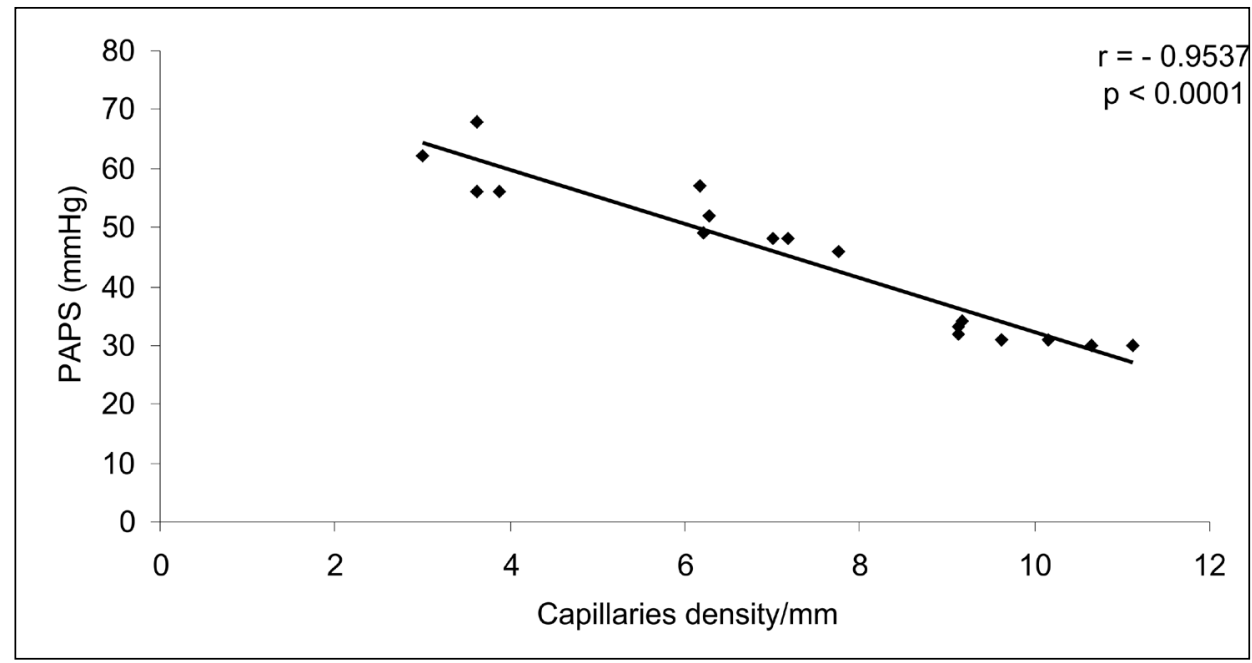

FIGURE 2. Pearson correlation between capillaries density and PASP in SSc patients 


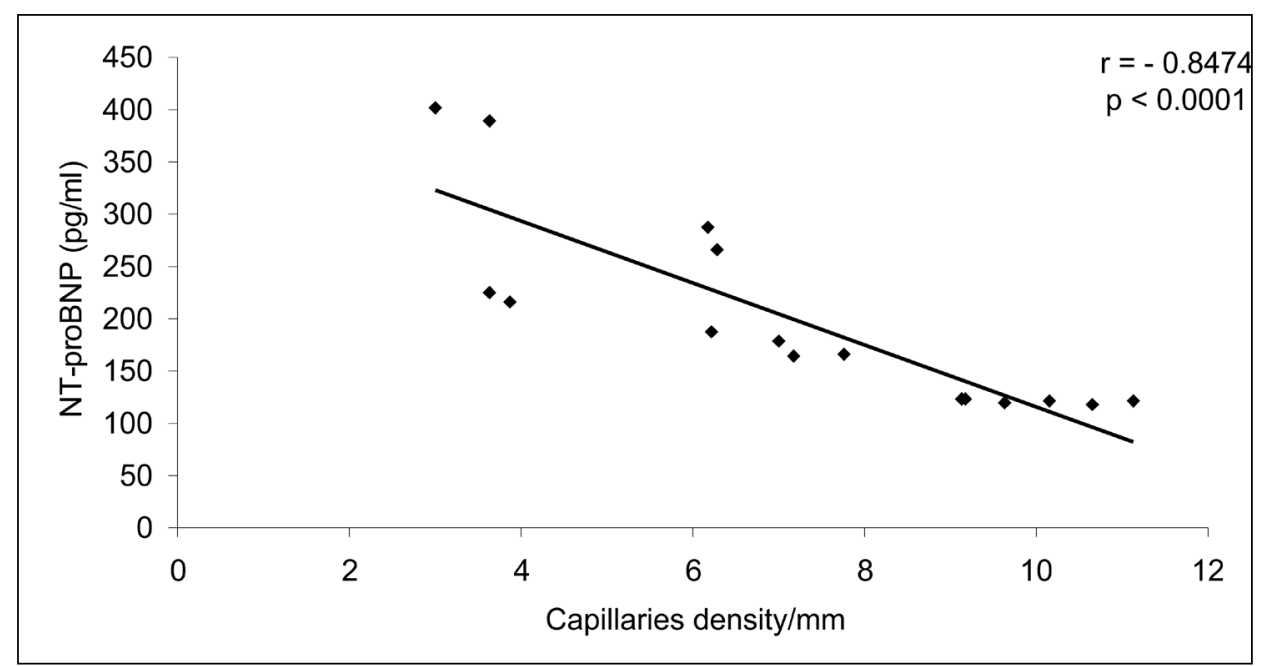

FIGURE 3. Pearson correlation between capillaries density and NT-proBNP in SSc patients

stitial lung disease, and peripheral vascular disease $(23,24)$.

The present study found a negative correlation between capillary density reduction and increasing PASP $(p<0.0001)$. The PASP values were chosen to characterize the presence of $\mathrm{PAH}$ in the studied SSc patients. As the capillaroscopic pattern worsened, PASP increased. Ong et al. reported that SSc patients with PAH had a significant reduction in capillaries density compared with SSc patients without this comorbidity (25). Hofstee et al. showed that capillary density was lower in SSc patients with PAH than in SSc patients without PAH and was correlated with the severity of PAH (14). In a small study of $24 \mathrm{SSc}$ patients, Riccieri et al. found significant correlations between mean pulmonary artery pressure values, the nailfold videocapillaroscopy score, and the avascular areas score (26). Another study showed that severe skin telangiectasias were independently associated with late capillaroscopic pattern (OR 4.84 [95\% CI 1.32-26.19]; $\mathrm{p}=0.018$ ) and PAH (OR 12.60 [95\%CI 1.68-94.53]; $\mathrm{p}=0.014)$ (17). Emrani et al. revealed the inverse correlation between the decrease in capillaries density and presence of PAH (27).

Several features of PAH have been identified in the two SSc subsets. Thus, dcSSc patients were younger, had a shorter mean length evolution, and a reduced $\mathrm{FVC}$ than $\mathrm{lcSSc}$ patients.

In dcSSc, PAH is always associated with significant interstitial lung disease, reflected by reducing FVC (2). A significant correlation between FVC, as a measure of pulmonary fibrosis, and capillary density has been described in dcSSc patients. Bredemeier et al. showed that ground-glass opacities on high-resolution computed tomography were correlated with a higher avascular score on nailfold capillaroscopy (28). In a study of 92 patients with dcSSc, Sato et al. reported significant correlations between vascular deletion score, $\mathrm{PAH}$, and interstitial lung disease (29).

NT-proBNP is a novel biomarker associated with PAH. NT-proBNP is a 76-amino acid N-terminal inactive protein that is cleaved from proBNP. It has been shown to be of great benefit in identifying SSc patients with PAH. At a cut-off value of $125 \mathrm{ng} / \mathrm{L}$, NT-proBNP is strongly and independently associated with three- and five-year mortality in SSc patients and may represent a potential predictor of mortality in this population (12). In our study, we identified a significant positive correlation between NT-proBNP and PASP. In a study by Thakkar et al. on SSc patients, NT-proBNP was highest in the group of SSc patients with PAH compared with SSc patients without PAH, and higher in the risk group compared with controls. The authors demonstrated a positive correlation between NT-proBNP and PASP (30). In the PHAROS registry, it was shown that NT-proBNP may be more useful than BNP in the detection and monitoring of PAH in SSc patients (31). Due to a good correlation between NT-proBNP values and pulmonary artery pressure, Thakkar et al. proposed that NT-proBNP and pulmonary functional tests should be markers of SSc-related PAH (32). Several studies demonstrated the correlation between NT-proBNP and PASP in SSc patients $(33,34,35$, 36).

The NT-proBNP values were significantly correlated with reduced capillary density and with the se- 
verity of nailfold capillaroscopic pattern. Higher values of NT-proBNP were more common in patients with extended avascular areas (37). Markusse et al. identified that a more severe nailfold capillaroscopic pattern was associated with the risk for interstitial lung disease $(\mathrm{OR}=1.34,95 \%$ CI 1.04-1.74), reduced DLCO (OR=1.52, 95\% CI 1.17-1.98), increased PASP (OR $=2.33$, 95\% CI 1.01-5.39), and increased NT-proBNP (OR=1.70, 95\% CI 1.09-2.64) (38). In the study performed by Stefanovic Neskovic et al., statistically significant BNP increases were observed with the increase in capillaroscopic pattern severity (39).

The small number of SSc patients, along with the retrospective nature of the study, limit our results. A multidisciplinary assessment comprising of the rheumatologist, cardiologist, and pulmonologist is essential for the appropriate management of SSc patients with PAH.

\section{CONCLUSIONS}

1. Structural changes in the nailfold microcirculation identified by nailfold capillaroscopy are simi- lar to those in other microvascular territories, including the pulmonary circulation. Thus, nailfold capillary density may represent "a mirror" of pulmonary circulation in SSc patients.

2. The presence of PAH may be assessed by cardiac ultrasound, right ventricular catheterization, or by serum NT-proBNP.

3. In SSc patients, significant correlations have been identified between NT-proBNP, PASP, and nailfold capillaries density, and between nailfold capillaries density and PASP.

4. Several features of PAH have been identified in the two SSc subsets. dcSSc patients were younger, had a shorter mean length of disease progression, and a reduced FVC compared to lcSSc patients. In $\mathrm{lcSSc}, \mathrm{PAH}$ is usually isolated, whereas in $\mathrm{dcSSc}$, it is associated with interstitial lung disease.

5. NT-proBNP and cardiac ultrasonography represent non-invasive markers for early identification of PAH in SSc patients with an advanced capillaroscopic pattern.

Conflict of interest: none declared Financial support: none declared

\section{REFERENCES}

1. Bellando-Randone S, Guiducci S, Matucci-Cerinic M.. Very early diagnosis of systemic sclerosis. Pol Arch Med Wewn 2012;122 (Suppl 1): 18-23

2. Hachulla E, Czirjak L (ed.). Textbook on Systemic Sclerosis. London: BMJ Publishing Group, 2013

3. Souza EJ, Kayser C. Nailfold capillaroscopy: relevance to the practice of rheumatology. Rev Bras Reumatol 2015. http://dx.doi. org/10.1016/j.rbre.2014.09.005

4. Grassi W, Core P, Carlino G et al. Labial capillary microscopy in systemic sclerosis. Ann Rheum Dis 1993; 52(8): 564-569

5. Cutolo M, Sulli A, Secchi ME et al. Nailfold capillaroscopy is useful for the diagnosis and follow-up of autoimmune rheumatic diseases. A future tool for the analysis of microvascular heart involvement? Rheumatology 2006; 45 (suppl 4): iv43-iv46

6. Cutolo M, Sulli A, Pizzorni C, Accardo S. Nailfold videocapillaroscopy assessment of microvascular damage in systemic sclerosis J Rheumatol 2000; 27(1): 155-60

7. Cutolo M. Atlas of capillaroscopy in rheumatic diseases. Milano: Elsevier, 2011

8. Mukerjee D, St George D, Coleiro B et al. Prevalence and outcome in systemic sclerosis associated pulmonary arterial hypertension: application of a registry approach. Ann Rheum Dis 2003; 62: 108893

9. Muangchan C. Canadian Scleroderma Research G, Baron M, Pope $\mathrm{J}$. The $15 \%$ rule in scleroderma: the frequency of severe organ complications in systemic sclerosis. A systematic review. J Rheumatol 2013; 40(9): 1545-56

10. Steen V, Medsger TA. Predictors of isolated pulmonary hypertension in patients with systemic sclerosis and limited cutaneous involvement. Arthritis Rheum 2003; 48: 516-522

11. Coral-Alvorado $P$, Rojas-Villarraga $A$, Latorre $M C$ et al. Risk factors associated with pulmonary arterial hypertension in Colombian patients with systematic sclerosis: review of the literature. $J$ Rheumatol 2008; 35: 244-250

12. Allanore $\mathrm{Y}, \mathrm{K}$ Komocsi $\mathrm{A}$, Vettori $\mathrm{S}$ et al. $\mathrm{N}$-terminal pro-brain natriuretic peptide is a strong predictor of mortality in systemic sclerosis. Int J Cardiol 2016; 223: 385-389

13. Caramaschi P, Canestrinini S, Martinelli N et al. Scleroderma patients nailfold videocapillaroscopic patterns are associated with disease subset and disease severity. Rheumatology 2007; 46: 1566-9

14. Hofstee HM, Vonk Noordegraaf A, Voskuyl AE et al. Nailfold capillary density is associated with the presence and severity of pulmonary arterial hypertension in systemic sclerosis. Ann Rheum Dis 2009; 68: 191-5

15. Steen VD, Medsger TA jr. Severe organ involvement in systemic sclerosis with diffuse scleroderma. Arthritis Rheum 2000; 43(11): 2437-2444

16. van den Hoogen F, Khanna D,Fransen J et al. 2013 Classification Criteria for Systemic Sclerosis: an American College of Rheumatology/European League against Rheumatism collaborative initiative. Ann Rheum Dis 2013; 72(11): 1747-55

17. Galie N, Humbert M, Vachiery JL et al. 2015 ESC/ERS Guidelines for the diagnosis and treatment of pulmonary hypertension: The Joint Task Force for the Diagnosis and Treatment of Pulmonary Hypertension of the European Society of Cardiology (ESC) and the European Respiratory Society (ERS): Endorsed by: Association for European Paediatric and Congenital Cardiology (AEPC), International Society for Heart and Lung Transplantation (ISHLT). European Heart Journal 2016; 37(1): 67-119

18. Trojanowska M. Pulmonary hypertension associated with scleroderma and connective tissue disease: Potential molecular and cellular targets. Advances in Pulmonary Hypertension 2017; 16 (2): 61-67

19. Romanowska-Próchnicka K, Walczyk M, Olesińska M. Recognizing systemic sclerosis: comparative analysis of various sets of classification criteria. Reumatologia 2016; 54(6): 296-305

20. Pattanaik D, Brown M, Postlethwaite AE. Vascular involvement in systemic sclerosis (scleroderma). J Inflamm Res 2011; 4: 105-125 
21. Cutolo M, Smith V. State of the art on nailfold capillaroscopy: a reliable diagnostic tool and putative biomarker in rheumatology? Rheumatology 2013; 52: 1933-1940

22. Matucci-Cerinic M, Kahaleh B, Wigley FM. Review: evidence that systemic sclerosis is a vascular disease. Arthritis Rheum. 2013; 65(8):1953-62

23. Cutolo M, Sulli A, Smith V. Assessing microvascular changes in systemic sclerosis diagnosis and management. Nat Rev Rheumatol 2010; 6: 578-587

24. Sulli A, Pizzorni C, Smith V et al. Timing of transition between capillaroscopic patterns in systemic sclerosis. Arthritis Rheum. 2012; 64(3): 821-5

25. Ong YY, Nikoloutsopoulos T, Bond CP et al. Decreased nailfold capillary density in limited scleroderma with pulmonary hypertension. Asian Pac J Allergy Immunol 1998; 16: 81-6

26. Riccieri V, Vasile M, lannace $N$ et al. Systemic sclerosis patients with and without pulmonary arterial hypertension: a nailfold capillaroscopy study Rheumatology 2013; 52: 1525-1528

27. Emrani Z, Karbalaie A, Fatemi A et al. Capillary density: An important parameter in nailfold capillaroscopy Microvasc Res 2017; 109: 7-18

28. Bredemeier M, Xavier RM, Capobianco KG et al. Nailfold capillary microscopy can suggest pulmonary disease activity in systemic sclerosis. J Rheumatol 2004; 31(2): 286-94

29. Sato LT, Kayser C, Andrade LE. Nailfold capillaroscopy abnormalities correlate with cutaneous and visceral involvement in systemic sclerosis patients. Acta Reumatol Port 2009; 34(2A): $219-27$

30. Thakkar V, Stevens WM, Prior D et al. N-terminal pro-brain natriuretic peptide in a novel screening algorithm for pulmonary arterial hypertension in systemic sclerosis: a case-control study. Arthritis Res Ther 2012; 14(3): R143
31. Chung L, Fairchild RM, Furst DE et al. Utility of B-type natriuretic peptides in the assessment of patients with systemic sclerosisassociated pulmonary hypertension in the PHAROS registry. Clin Exp Rheumatol 2017; 35 Suppl 106(4): 106-113

32. Thakkar V, Stevens $\mathrm{W}$, Prior $\mathrm{D}$ et al. The inclusion of $\mathrm{N}$-terminal pro-brain natriuretic peptide in a sensitive screening strategy for systemic sclerosis-related pulmonary arterial hypertension: a cohort study. Arthritis Res Ther 2013; 15(6): R193

33. Cavagna L, Caporali R, Klersy $\mathrm{C}$ et al. Comparison of brain natriuretic peptide (BNP) and NT-proBNP in screening for pulmonary arterial hypertension in patients with systemic sclerosis. J Rheumatol 2010; 37(10): 2064-70

34. Le Pavec J, Humbert M, Mouthon L, Hassoun PM. Systemic sclerosis-associated pulmonary arterial hypertension. Am J Respir Crit Care Med 2010; 181(12): 1285-93

35. Coghlan JG, Denton CP, Grunig E et al. Evidence-based detection of pulmonary arterial hypertension in systemic sclerosis: the DETECT study Ann Rheum Dis 2013; 00: 1-10

36. Hoffmann-Vold AM, Midtvedt O, Garen T et al. Assessment of NT-ProBNP as a potential marker for pulmonary hypertension in systemic sclerosis: Data from a large, prospective and unselected patient cohort [abstract]. Arthritis Rheumatol 2015; 67 (suppl 10)

37. Teixeira L, Cordeiro I, Sousa S et al. Nailfold capillaroscopy findings in scleroderma patients - prognostic implications. Ann Rheum Dis 2016; 75: 754

38. Markusse IM, Meijs J, de Boer B et al. Predicting cardiopulmonary involvement in patients with systemic sclerosis: complementary value of nailfold videocapillaroscopy patterns and disease-specific autoantibodies. Rheumatology 2017; 56 (7): 1081-1088

39. Stefanovic Neskovic J, Ristic A, Petronijevic M et al. B-type natriuretic peptide as a marker of different forms of systemic sclerosis. J Med Biochem 2018; 37: 406-414 